\title{
RECENT ADVANCES AND FUTURE PROSPECTS OF NON-INVASIVE INSULIN DELIVERY SYSTEMS
}

\author{
MOAWIA M. AL-TABAKHA*, SALWA SAMI MUBARAK, BALSAM SAAD AZEEZ
}

Department of Pharmaceutical Sciences, College of Pharmacy and Health Sciences, Ajman University, Ajman, UAE

Email: m.altabakha@ajman.ac.ae

Received: 30 Oct 2018, Revised and Accepted: 12 Feb 2019

\begin{abstract}
Non-invasive insulin delivery systems have been of global interest. The goal of many studies was to optimize suitable delivery formulation capable of producing comparable insulin bioavailability and safety that match or supersedes conventional delivery by the invasive subcutaneous (SC) injections. Historically, Pfizer marketed the first insulin inhaler Exubera ${ }^{\circledR}$ in 2006 which was later retracted from the market before completing the two years. In recent years, Afrezza ${ }^{\circledR}$, a new inhalator, and Oral-Lyn ${ }^{\mathrm{m}}$, a buccal spray, have been introduced to the market. While Afrezza ${ }^{\circledR}$ lost the marketing and distribution support from Sanofi, Oral-Lyn ${ }^{\text {TM }}$ have not secured US Food and Drug Administration (FDA) and the European Medicines Agency (EMA) marketing approval yet. Different technologies to improve insulin's permeation and absorption through different routes are in the pipelines. This review discusses several non-invasive strategies that have been appropriately tested and duly approved by the FDA. Other delivery systems are in different phases of development, ranging from in vitro studies to phase 3 clinical trials, providing indications towards the prospects of next-generation delivery systems. This review covers studies published in the past 10 y using Scopus, Clinicaltrials.gov, PubMed and Google scholar databases. The outcomes of this review indicate that the door is still open for more innovative, efficient and convenient non-invasive insulin delivery systems than currently available which may take several years before we can see a game changer in the market.
\end{abstract}

Keywords: Oral insulin, Afrezza ${ }^{\circledR}$, Oral-Lyn $^{\mathrm{Tm}}$, Insulin patches, Needle-free Injections, Insulin jet injectors

(C) 2019 The Authors. Published by Innovare Academic Sciences Pvt Ltd. This is an open access article under the CC BY license (http://creativecommons.org/licenses/by/4.0/) DOI: http://dx.doi.org/10.22159/ijap.2019v11i2.31338

\section{INTRODUCTION}

The prevalence of diabetes mellitus (DM) which is a metabolic disorder is dramatically increasing worldwide, making it one of the major causes of morbidity and mortality $[1,2]$. In Saudi Arabia, the use of antidiabetic medication for type 2 DM patients represents the fourth most utilized medication in the country indicating the widespread of this condition [3]. All diabetic patients may require insulin administration at some point of time. Type 2 DM uncontrolled by oral hypoglycaemic drugs, may require having insulin added to their therapy regimen [4]. Controlling diabetes with subcutaneous (SC) insulin delivery requires multiple daily injections which are considered inconvenient for many diabetic patients since it can be accompanied by pain, lipodystrophy, tissue damage and allergic reactions at the site of injection $[5,6]$. Moreover, the method of the administration itself makes it inconvenient to perform in public places.

It has been of interest to academic and pharmaceutical industry researchers, especially over the past three decades, to look up for an alternative to SC insulin injection to achieve better patient compliance and disease complications control, hence avoiding associated morbidity and mortality rates [7]. Unfortunately, insulin has very poor oral bioavailability as it is easily degraded by proteolytic enzymes in the gastrointestinal tract and the enterocytes of the intestine as well as various peptidases at the brush-border membrane [8]. The search for non-invasive delivery systems for insulin by optimizing the delivery of insulin had not produced a product that can substitute the traditional method of injecting insulin subcutaneously. Despite this, there are only two products for the non-invasive delivery of insulin available in the market which are $A_{\text {frezza }}{ }^{\circledR}$, a pulmonary inhalation product and Oral-Lyn ${ }^{\text {mM }}$, a buccal spray. Therefore, the purpose of this review is to systematically collect and analyse available literature related to noninvasive insulin delivery systems to identify both current systems and those that are in the pipeline and draw a conclusion regarding the future trends in non-invasive systems.

\section{Systematic literature review}

Studies and articles that were published throughout the past $10 \mathrm{y}$ were collected from the following databases: Scopus,
Clinicaltrials.gov, PubMed and Google Scholar. These databases can provide access to peer-reviewed literature and information about medical studies in human volunteers. Scopus covers a different array of research fields including science, health and medicine and contains over 22,800 journal titles from over 5,000 publishers. Clinicaltrials.gov is a resource provided by the U. S. National Library of Medicine and contains over 273,004 research studies in the USA and in 203 countries. PubMed comprises more than 28 million citations for biomedical literature from MEDLINE, life science journals, and online books. Google Scholar indexes online reviewed journals and books, conference proceedings, theses, patents and other scholarly literature from diverse disciplines. Clinicaltrials.gov, PubMed, and Google Scholar are free to search databases.

Each database used provides tools for searching and filtration of outcomes. On Scopus, and PubMed, searching was done using the following terms/sentences: "insulin drug delivery" and "insulin delivery systems" with limitations to articles published in the past $10 \mathrm{y}$ in the English language. Because Google Scholar tends to produce a very large number of articles with no option to limit the type of the articles, the advance search was used to limit them to the keywords present in the article titles only. In addition, Clinicaltrials.gov was used to search for the same keywords under "other terms" field of advanced search. Also, the advanced search was used to implement the years limit to acquire results from interventional trials only.

Publications from literature review were additionally filtered in all databases used depending on the abstracts and article titles to exclude results limited to invasive routes of insulin administration, artificial pancreas, new antidiabetic drugs and glucose monitoring techniques.

\section{Database and outcomes}

The number of hits from different databases used are shown in fig. 1. The lowest number of hits was provided for Google Scholar ( 81 for "insulin drug delivery" and 108 hits for "insulin delivery systems") and the largest number of hits was obtained with Scopus (1928 for "insulin drug delivery" and 1892 for "insulin delivery systems"). 


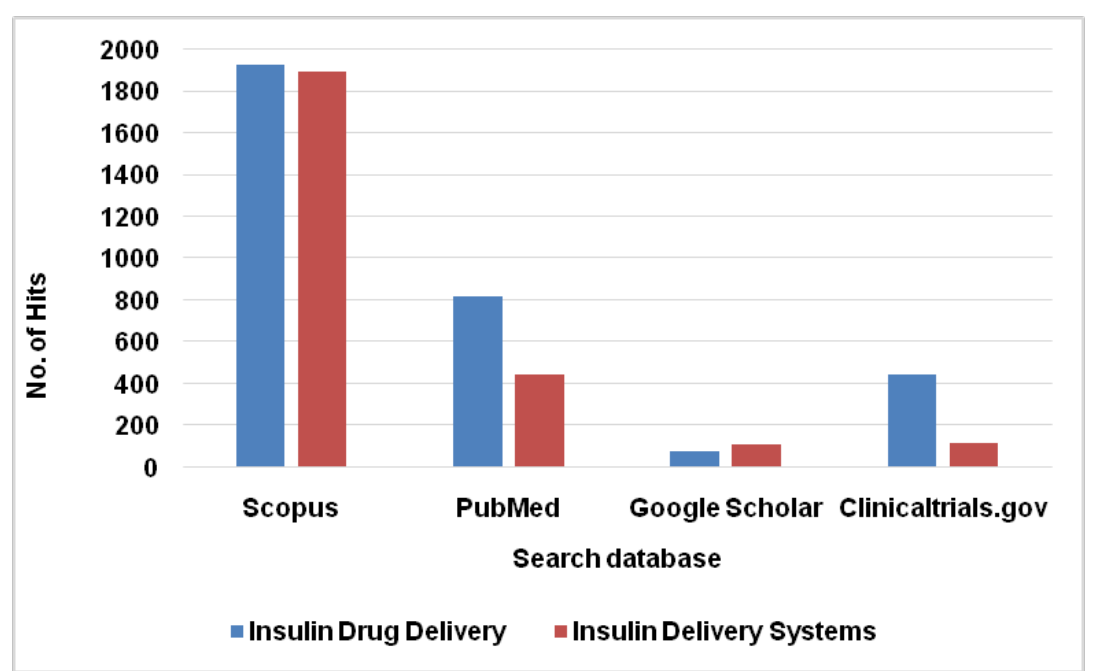

Fig. 1: Number of hits obtained by using scopus, PubMed, google scholar and Clinicaltrials.gov with the keywords "insulin drug delivery" and "insulin delivery systems" databases, for online publications since 2008

Reviews, clinical trials and research articles used to collect information about non-invasive insulin delivery which was categorized according to the route of administration.

\section{Oral delivery}

Oral administration of insulin represents the most convenient route and attractive approach to developing systems that are capable of protecting insulin and enhancing its absorption [9]. Insulin is a peptide of almost negligible bioavailability (1\%) when orally administered as a conventional solution, due to degradation by gastrointestinal protease enzymes, gastric acid inactivation and low intestinal membrane permeability [6]. Therefore, it has always been a challenge to establish oral formulation using different methods and technologies aiming to yield efficient and safe products. The following subsections present several approaches:

\section{Nanoparticles (NPs)}

They are used mainly to enhance protection and targeting of the loaded drugs. An in vivo study on $\mathrm{N}$-trimethyl chitosan chloride nanoparticles (TMC-NPs) loaded with insulin found that upon coating NPs with FQSIYPpIK peptide (FQS), the intestinal epithelium cellular uptake of insulin increased more than uncoated NPs [10]. FQSIYPpIK (FQS) peptide exhibits high affinity to integrin $\alpha v \beta 3$ receptor which is a transmembrane glycoprotein. FQS-NPs promoted serum insulin concentrations, increasing slowly to a maximum reached after $4 \mathrm{~h}$ of oral administrations, with a bioavailability of $7.58 \%$ which is 25 times higher than oral free insulin of $0.3 \%$ bioavailability.

Another NPs system in which insulin was encapsulated within polymeric chitosan chains and covalently cross-linked with the chains of polyethylene glycol (PEG) produced rigid and ultrasmall $(<15 \mathrm{~nm})$ stable NPs over a wide $\mathrm{pH}$ range. These produced NPs were further conjugated to PenetraMax, a cell-penetrating peptide developed by sequence modification of penetration [11]. This technique is still within in vitro testing but promising to be adopted as a method for protein, peptide and vaccines delivery since this model as water-in-oil (W/O) nanoemulsion containing insulin achieved an increase in insulin translocation across the Caco- 2 cell line by $18 \%$ compared to oral insulin solution.

One in vivo study used diabetic adult male Wister rats to administer low molecular weight chitosan nanoparticles in which sodium lauryl sulfate is used as a micellar former that is concurrent with chitosaninsulin complex [12]. The results showed acidic $\mathrm{pH}$ stability in which only $10 \%$ of loaded insulin was released and inactivated after $1 \mathrm{~h}$ incubation period. The preparation showed a significant lowering of blood glucose levels (BGLs), however in comparison to the SC insulin this reduction was modest.
A lipid bio-nanoparticle technology for insulin encapsulation into hepatic directed vesicles (HDV-I) was studied in a single-blind, placebo-controlled trial. HDV-I comprised of hepatocyte targeting molecule (HTM) biotin-phosphatidylethanolamine with $1.1 \mathrm{mg}$ of HDV-phospholipid filled in gelatin capsules for administration 30 min prior to each standardized meal. The postprandial plasma glucose (PPG) and incremental PPG area under the concentrationtime curve were significantly lower compared to placebo [13] which is clinically relevant, but a linear dose-response was not observed. The treatments did not result in any untoward effects such as hypoglycemic episodes, but a long-term randomized clinical trial is required.

Nanocarrier could be a biopolymer characterized by biodegradation, high stability and binding capacity for a loaded drug, no antigenic reaction with the immune system and low toxicity [14]. The use of egg albumin nanoparticles for oral insulin administration prepared by microemulsion crosslinking method has been investigated in vitro. Egg albumin nanoparticles released more insulin at neutral $\mathrm{pH}$ compared to acidic or alkaline $\mathrm{pH}$ and this was related to their swelling and therefore the diffusibility of insulin. These prepared nanoparticles could be further investigated in vivo in a future study.

Another interesting nanoparticle system of imprinted insulin and insulin bound islets was developed using the precipitation polymerization method for oral delivery. Biomimetic molecular imprinted polymers (MIPs) have robust physical structures, biocompatibility, and loading capabilities and can serve as a carrier for proteins and peptides including insulin [15]. In vivo investigation using male Wistar rats proved the efficacy of MIPs in targeting delivery of insulin by $\mathrm{pH}$-sensitive release and variant degree of glucose level reduction based on ratio and type polymers used. The bioavailability of insulin from the orally administered nanoparticles was comparable to the SC insulin injections when the insulin dose was 50:1 respectively.

Another biomimetic technique involves the use of vitamins (thiamine and niacin) as a ligand decorating liposomes nanocarriers provide targeting to enterocytes since wide receptors for these ligands are distributed in intestinal epithelium enhance penetration and delivery of insulin [16]. Upon comparison with SC insulin injection, vitamin decorated liposomes showed a mild hypoglycaemic effect that lasts for nearly $12 \mathrm{~h}$ and an oral bioavailability of $2.5 \%$.

\section{Lipopeptide}

A bacillus lipopeptide product called surfactin promotes resistance to digestion by trypsin and pepsin with amphiphilic nature that gives it a strong biosurfactant action [6]. In vivo studies showed that the combination can produce a hypoglycaemic effect with relative 
bioavailability $12.48 \%$ for diabetic mice and $5.97 \%$ for non-diabetic dogs. Surfactin in acidic media co-precipitates with insulin and provide degradation protection, while in basic media the coprecipitate will re-dissolve and a heteropolymer is formed upon surfactin-insulin binding which in turn promotes intestinal absorption.

\section{Insulin chewing gum}

Chewing gum has been used over the world to deliver different drugs and molecules like aspirin, dimenhydrinate, nicotine, and others. Insulin-containing chewing gum is suggested in which mastication drug would be introduced to saliva for absorption through the oral mucosa. Vitamin B12 is a large molecule requiring an intrinsic factor to be transported and absorbed such as haptocorrin, which is also present in saliva. This has been utilized to improve insulin absorption from the oral cavity. Vitamin B12 was used to coat insulin in the chewing gum to facilitate its absorption [17]. In vivo trials on diabetic rats reported a blood glucose reduction when introducing vitamin B12-Insulin conjugate higher than free insulin as indicated by a 4.7 -fold reduction in the area under the area under the blood glucose curve. This formulation had a fast onset, fewer side effects, improved bioavailability and ease of administration while on the other hand it might be associated with flatulence, diarrhoea, facial muscle pain and enamel dentures.

\section{Liposomes}

Liposomes have been indicated for drug delivery through different routes showing an enhanced bioavailability especially for orally administered lipids and proteins $[18,19]$. One study suggested the use of liposomes containing phospholipid/botanical cholesterol analogs for insulin delivery to improve gastrointestinal stability compared to phospholipid/cholesterol liposome. Human recombinant insulin was loaded into liposomes containing $\beta$ sitosterol, stigmasterol, lanosterol, and ergosterol and tested in vitro and in vivo for comparison. Ergosterol liposomes showed higher stability and oral bioavailability than other formulations. The optimal compositions were $1: 4$ and $1: 6$ molar ratios of phospholipid/ergosterol resulting in constant BGL reduction of $50 \%$ over $3 \mathrm{~h}$ in normal rats [18].

Another liposomes model of phospholipid/sodium glycocholate loaded with recombinant human insulin has been suggested. Sodium glycocholate is a bile salt has the advantages of protease enzymes inhibition, permeation enhancement, and low cytotoxicity. In vitro tests showed a high entrapment efficiency of insulin (30\%) and upon comparison to other bile salts like sodium deoxycholate and sodium taurocholate, sodium glycocholate liposomes showed enhanced protection of inulin and hence has the potential for providing improved insulin bioavailability [19] and therefore, further in vivo tests will be needed.

Folic acid (FA) was also proposed as a targeting technique for insulin-loaded liposomes towards FA receptors which are frequently expressed over the GI tract. This will increase the uptake of loaded bioactive insulin [20]. In vivo testing on diabetic rats showed that FA-Insulin layersomes, prepared by core insulin liposomes coated with layer-by-layer oppositely charged polyelectrolytes and FA added to provide ligand-receptor targeting, produced $20 \%$ relative bioavailability compared to SC insulin and a reduction in BGL of 78\% over $6 \mathrm{~h}$ period.

\section{Hydrogel}

A hydrogel is a three-dimensional hydrophilic polymer that may offer protection of loaded protein from digestive enzymes, extending residence time in the intestine and enhancing paracellular transport of proteins [21]. A hydrogel that is poly (methacrylic acid-graft-ethylene glycol) P(MAA-g-EG) microparticles used as a carrier for insulin and insulin-transferrin conjugate was evaluated for insulin permeability using Caco-2/HT29-MTX as the cellular model. Results revealed that the conjugation of insulin with transferrin by its own increased insulin transport 7-folds compared to insulin alone because transferrin directed insulin to intestinal epithelium through transferrin receptors on these cells. The presence of P(MAA-g-EG) microparticles induced a 14-folds increase in conjugate transportation.
An in vivo study on the use of polymethacrylic acid-co-N-vinyl pyrrolidine P(MAA-co-NVP) hydrogel for oral protein transportation using insulin as a model drug showed a loading efficiency greater than $85 \%$ [22]. At acidic $\mathrm{pH}$, no insulin release was detected while in neutral and basic $\mathrm{pH}$ rapid release occurred. Prepared copolymeric hydrogels composed of $\mathrm{N}$-vinylcaprolactam and methacrylic acid monomers by free radical polymerization for oral delivery of insulin. The preparation showed an entrapment ratio of $52 \%$ for insulin and the capability to protect it from the acidic condition while swelling and releasing insulin in the basic media, therefore offering an opportunity for oral delivery of insulin [23].

A natural hydrogel comprised of calcium pectinate beads (microbeads size ranging between 180 and $220 \mu \mathrm{m}$ ) containing insulin was prepared by ionotropic gelation technique with the aid of airflow from an air compressor. Insulin release was dependent on the phosphate, calcium and pectin concentrations in the preparations of the microbeads and the results suggest them to be promising carriers for oral delivery of proteins, but further in vivo evaluation is recommended [24].

\section{Microparticles}

In one study microparticles were prepared by consecutive adsorption of oppositely charged chitosan (Ch) and dextran sulfate (DS) into insulin-DS microaggregate and were subsequently characterized for biocompatibility and biodegradability. This produced a highly stable microparticles in acidic $\mathrm{pH}$ with no drug release until reaching the neutral $\mathrm{pH}$ when insulin release starts and by $\mathrm{pH}$ of 8.0 all insulin would be released [25]. Ch has mucoadhesive and epithelium tight junctions opening properties. Hence insulin would be protected against denaturation and increased paracellular adsorption would result. In vivo studies on healthy Chinchilla male rabbits and diabetic male Wistar rats showed hypoglycaemic effects and relative bioavailability of $10.7 \%$ in comparison to SC insulin administration.

\section{Oral capsules}

Many studies were performed on chitosan due to its mucoadhesive property and biodegradability. Insulin oral capsules with the size of about $2.5 \mathrm{~mm}$ were suggested as potential carriers for oral insulin following an in vivo investigation on diabetic rats. The capsules were made of chitosan, alginate, and insulin which demonstrated that by increasing the insulin dose from 20 to $40 \mathrm{IU}$, glucose level decreased further and sharper from $33.7 \%$ to $51.7 \%$ of the initial value after two hours of administration [26].

Chitosan was also used in phytic acid-chitosan capsule preparation with the size of $1.68 \mathrm{~mm}$ for orally administered insulin. Phytic acid is naturally occurring composed of many anions that are crosslinked with cations of chitosan forming a stable medium for insulin encapsulation [27]. In vivo test on diabetic rats showed that orally administered capsule of $40 \mathrm{IU} / \mathrm{Kg}$ insulin produced more than $50 \%$ reduction on BGL sustained for more than $24 \mathrm{~h}$, compared with SC insulin administration which resulted in sharp blood glucose reduction $84.3 \%$ in $1 \mathrm{~h}$ and gradually vanished within $12 \mathrm{~h}$.

Another promising enterically coated insulin capsules were studied, whereby they would dissolve in the intestine and release the mucoadhesive devices (patches) that direct the release of insulin in one direction [28]. The devices were prepared by compressing 1:1:2 ratio of Eudragit ${ }^{\circledR}$ E PO, pectin and sodium carboxymethylcellulose polymers, and permeability enhancer (dimethyl palmitoyl ammonio propane sulfonate). Biopsy punches were used to obtain $2 \mathrm{~mm}$ discs from the device for encapsulation into size 9 capsules that were later enterically coated with Eudragit ${ }^{\circledR} 100$. In vivo tests on male Wistar diabetic and healthy rats show that drug release started at or greater than $\mathrm{pH}$ of 5 providing a glucose level reduction of 30 to $33 \%$ sustained for up to $8 \mathrm{~h}$. For human size capsules, a new generation of ready to use delayed release vegetable capsules could be used [29].

Enteric microspheres (EMS) in which insulin was loaded, were suggested for oral route formulation. Insulin-loaded EMS was prepared by $01 / 02$ emulsion solvent evaporation method. The microspheres were composed of hydroxypropyl methylcellulose acetate succinate to provide enteric properties, bacitracin (protease inhibitor) to prevent 
cleavage and unfolding of insulin and sodium oleate for absorption enhancement. EMS formulations were administered orally into male Wistar diabetic rats after being filled into gelatine capsule. Measurements showed no reduction in BGL within the first $2 \mathrm{~h}$ then intense reduction through $5 \mathrm{~h}$ and beyond [30].

\section{Microemulsions}

Microemulsions have been used for oral delivery of proteins because of protection and enhanced absorption. The method is characterized by thermodynamic stability and is formed by mixing water, oil, and surfactant [31]. Water in oil microemulsion into which insulinchitosan nanoparticles were loaded was evaluated. Oral administration of this microemulsion to diabetic male Wistar rats resulted in significant blood glucose reduction that was maintained over $8 \mathrm{~h}$ period in comparison to SC inulin injection that causes rapid reduction for a period of $2 \mathrm{~h}$ only.

\section{Bucal delivery}

In one study buccal sprays were prepared by adding the proper amount of insulin stock solution to two different solutions of absorption enhancers, $5 \%$ azone and 3\% soybean lecithin (SLT) yielding a $40 \mathrm{IU}$ of insulin per $\mathrm{ml}$ of solutions. Rabbits were used in the study since their buccal mucosa is similar to humans by being non-keratinized. BGLs measurements showed total decreasing rates of $32.1 \%$ and $43.5 \%$ for sprays with Azone and STL respectively in comparison to a control group. The relative bioavailabilities in comparison to SC insulin were $16.7 \%$ and $22.6 \%$ for sprays with Azone and STL respectively [5]. These findings support the use of SLT to develop a marketed insulin spray.

A marketed Oral-Lyn ${ }^{\text {TM }}$ by Generex biotechnology (Toronto, Canada) represents the first buccal spray of metered insulin doses. The proprietary device "RapidMist" resembles Ventolin ${ }^{\text {TM }}$ inhaler is used to spray the liquid formulation of human insulin. Each puff from the spray contains 10 units of insulin and only one unit (i.e. 10\%) is absorbed into blood circulation [32]. Oral-Lyn ${ }^{\text {TM }}$ could be used for both type 1 and 2 DM with pharmacokinetic characteristics similar to rapid-acting insulin $[32,33]$. A trial assessed the use of Oral-Lyn ${ }^{\text {TM }}$ for 15 type 1 diabetic patients for dinner time along with regular SC insulin in the morning and lunch times indicated a well-tolerated spray of fast onset within 5-10 min. and with lesser hypoglycaemic episodes [33]. The product has not yet received the Food and Drug Administration (FDA) marketing approval but has been marketed in some countries like India, Ecuador, Lebanon, and Algeria.

Buccal mucosa is of low permeability compared to the sublingual area but is also characterized by tolerance to sensitizers, rich perfusion and absorbed drugs avoid the first-pass metabolism encouraging the use of transmucosal delivery of macromolecular agents like proteins and peptides [34]. Controlled insulin delivery through buccal mucosa required the rate-limiting step to be the vehicle rather than the epithelium, hence absorption enhancers should be co-administered [5].

\section{Pulmonary delivery}

Pulmonary delivery is widely implemented for macro and micro drugs delivery due to the large alveolar surface area of $70 \mathrm{~m}^{2}$ to $140 \mathrm{~m}^{2}$, thin epithelial layer and the short distance between epithelium and alveolar blood vessels that is beneficial for effective drug absorption $[35,36]$. Thus, delivery of insulin through lungs was the first method reported as an alternative to SC injections [4, 37].

\section{Carriers and absorption regulators}

Since the lungs are permeable naturally to macromolecules and have low enzyme metabolic activity, insulin formulations were modified to prolong the absorption via the pulmonary route. Crystallization is one of the processes that play a major role in enhancing drugs stability and release. Insulin microcrystal powder combined with lactose carrier was prepared using crystallization. Upon examination of the hypoglycaemic effect of the formulation on diabetic rats, it was found that the powder formulation controls BGLs more effectively compared to a solution [38]. The bioavailability of the formulation was $15 \%$ relative to insulin SC injection and this confirms that microcrystal insulin powder can be a promising dosage form for insulin delivery.
A nanoparticulate system was a recently explored method for the transmucosal delivery of pharmaceutical molecules for the different purpose. Nanoparticles improve the loading capacity of drugs due to its high surface to volume ratio. Additionally, they raise the absorption of drugs by reducing epithelial resistance to transport. By adding natural polymers like chitosan (CS) and chondroitin (CHS) 'CS/CHS nanoparticles' it is believed that the delivery of macromolecules through the lungs would improve. One study investigated the capacity of CS/CHS nanoparticles to release insulin for the respiratory delivery [39]. The results indicated the suitability of this application as the formulations remained stable at $4{ }^{\circ} \mathrm{C}$ for one month and had good insulin release, but further studies would be needed to establish the real potential of their application.

Thiolated polymers 'thiomers' which contains thiol group provide higher adhesive properties than the regular nonthiolated mucoadhesive polymers [40]. This property is due to the formation of covalent bonds between the polymer and the mucosal layer by a disulfide bridge. Investigation of the thiolated microspheres containing insulin showed a greater reduction in BGLs on diabetic rabbits with maximum effect was observed at $6 \mathrm{~h}$ which was $31.23 \%$ of the initial glucose level compared to $75.25 \%$ for nonthiolated microspheres, proving thiolated microspheres ability as a useful drug vehicle for the nasal delivery system for insulin.

Excipients like cellulose derivatives: carboxymethyl cellulose, hydroxypropyl cellulose, and hydroxypropyl methylcellulose are usually used as binders for tablets manufacturing [41]. However, their effect on nasal absorption of peptides can be highly marked when formulated as powder and directly applied to the nasal mucosa. A study focusing on sodium carboxymethyl cellulose for the nasal delivery of insulin using rats showed improved absorption compared to insulin solution, which was attributed to a prolonged residence time of the powder formulation [42].

Also, tetradecyl-ß-D-maltoside (TDM) which is an alkyl glycoside surfactant consisting of 12-14 carbon alkyl chain can produce a transient enhancement in nasal permeability of peptides. It was mixed with fastacting and long-acting insulins and administered as nasal drops to hyperglycaemic rats. The results of the experiment revealed that fastacting insulin had rapid nasal absorption (Tmax = $15 \mathrm{~min}$ ), while the long-acting insulin, glargine insulin, had prolonged hypoglycaemic effect (Tmax $=60 \mathrm{~min}$ ) which support their use in nasal insulin products capable of producing sufficient control of BGLs [43].

Cell-penetrating peptides (CPPs) are a class of short polybasic and/or amphipathic peptides, having the ability to deliver numerous payloads of macromolecules across cell membranes, and have been recently studied for the nasal delivery of insulin [44]. Nasal absorption of insulin was successfully enhanced when formulated with novel penetratin analog "PenetraMax" with bioavailability reaching almost $100 \%$ relative to SC insulin administration without any toxicity or damage to the epithelial membrane.

Dextran is a class of complexly branched glucan that has various chain lengths, good water solubility and generally recognized as a safe excipient. To investigate the inhalation performance of dextran-peptide formulation, spray-dried insulin and dextran 10 (70\% insulin and 30\% D10) was compared to Exubera ${ }^{\circledR}$, a dry powder formulation of insulin previously approved and marketed for clinical use, in pure-bred female beagle dogs [45]. Both formulations produced similar bimodal particle size distribution, and the analysis of post insulin blood levels was comparable for both powders. Results suggest a promising path for dextran-peptide formulation for pulmonary delivery.

\section{Nasal gel}

In-situ gel system has conceivable application in enhancing delivery and controlled release of macromolecules in addition to its capacity to absorb an essential amount of biological fluids without dissolving its own structure. A thermosensitive in-situ gel was prepared by combining chitosan and polyvinyl alcohol for improving the nasal delivery of insulin [46]. The release of insulin from the gel was enough to maintain BGLs for six hours due to the bioadhesive characteristics of the gel. The maximum effect on lowering BGLs in normal rats was at $4 \mathrm{~h}$ compared to $1 \mathrm{~h}$ with SC insulin injection indicating prolonged insulin release that could result in lower hypoglycaemic episodes. The relative bioavailability from the 
gel was $8.8 \%$ which was better than the ineffective nasally administered insulin solution used for comparison. Nasal delivery of insulin is still challenged by the small surface area, metabolic activity, poor permeation and clearance [47].

\section{Insulin inhalation devices}

Exubera ${ }^{\circledR}$ was the first officially approved pulmonary insulin product by the FDA in $2006[48,49]$. The human insulin powder in blisters was taken using Exubera ${ }^{\circledR}$ inhaler (fig. 2-a) with the available $1 \mathrm{mg}$ and $3 \mathrm{mg}$ doses [4]. Unfortunately, due to the high cost and some concerns related to pulmonary functions, the device did not achieve its commercial targets and was withdrawn from the market by Pfizer Pharmaceuticals in 2007. An approved insulin inhalation system by FDA in June 2014 Afrezza ${ }^{\circledR}$ was developed by MannKind Corporation (California, United States) and marketed Sanofi (Paris, France) since January 2015. The system (fig. 2-b) overcame some disadvantages associated with the Exubera ${ }^{\circledR}$ such as the inhaler size and the flexibility and convenience of dosing and therefore was expected to have better performance in the market. The inhaled insulin is introduced in a pocket-size device where insulin is adsorbed to fumaryl diketopiperazine, and the microparticles are freeze-dried to form dry powder suitable for inhalation [50]. It needs approximately 10-14 min to reach maximum serum concentration and three hours for almost complete insulin absorption [51] and results in less hypoglycaemia [52]. The adverse effects associated with Afrezza ${ }^{\circledR}$ use are similar to Exubera ${ }^{\circledR}$ and include pulmonary function decline and a slight increase in lung cancer incidence although the latter effect was not substantiated [52]. Additional concerns included increased risk of acute bronchospasm and diabetic ketoacidosis was more common [51]. Afrezza ${ }^{\circledR}$ black box warning contraindicates its use in patients with chronic pulmonary diseases and mandates prescribers to assess patients' lungs and use spirometry which further limits its appeal. Sanofi had withdrawn from the agreement with the MannKind corporation to market Afrezza ${ }^{\circledR}$ and since July 2016, MannKind became the sole manufacturer and marketer. Mannkind believes that the challenges they have to deal with are limited by Sanofi's failure in marketing strategy including working with insurers and globalizing the product by filing for marketing approval in new regions. It is dubious if Mannkind will succeed when Sanofi had failed in gaining appropriate market share for this product in insulins market.

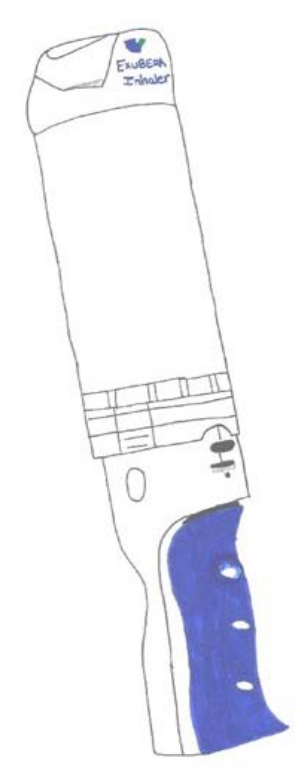

(a)

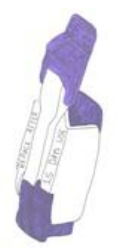

(b)

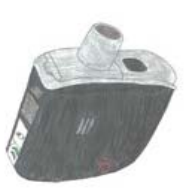

(c)
Fig. 2: Drawings of insulin inhalers depicting relative sizes to each other and representing (a) Exubera ${ }^{\circledR}$ in the extended position, (b) Afrezza ${ }^{\circledR}$ and (c) Dance $501^{\circledR}[48,51]$
With anticipated dose linearity, superior bioavailability and minimal side effects, a promising pulmonary device for is Dance $501^{\circledR}$ Dance Biopharm (Brisbane, California) is currently in phase 2 trial. The inhaler itself is based on technology developed and commercialized by Aerogen (Galway, Republic of Ireland). It is based on vibrating mesh technology combined with flow path to assist the cleaning of the drug reservoir. The small handheld device is battery operated (fig. 2-c) with an inhaler that delivers the liquid formulation of insulin into the lungs [53]. Despite the expected marginal advantages of this new insulin delivery system, it is unclear if the company will continue to pursue phase 3 trials since previously approved insulin inhalation systems did not demonstrate to be lucrative in the highly competitive market dominated by the various types of SC insulins.

\section{Transdermal delivery}

Transdermal delivery of insulin has been a point of interest as an alternative non-invasive administration method [54, 55]. However, stratum corneum which is the superficial layer of skin is a big challenge as it's not permeable to allow the transfer of a large molecule like insulin [56]. Therefore, many studies were performed to overcome this barrier and enhance skin permeation. This was examined through in vitro studies and animals and clinical trials. Latest penetration enhancement techniques are iontophoresis using electrical currents, sonophoresis using ultrasound waves, electroporation, microdermabrasion, micro-needles, chemical and biochemical enhancers [4].

\section{Sonophoresis}

In one study, the ultrasound waves with $40 \mathrm{kHz}$ frequency were applied to male albino Wistar rats to deliver insulin for $60 \mathrm{~min}$ in pulse mode [54]. The results of glucose-lowering were comparable to that of SC insulin injection indicating the effectiveness of the used technique. Despite the claimed success, the study did not provide details about the bioequivalence of insulin which seemed very low considering the high dose used with ultrasound ( $1 \mathrm{ml}$ of IU-100) in comparison to SC insulin $(0.25 \mathrm{IU} / \mathrm{Kg})$. Additionally, the air ultrasonic ceramic transducer producing the waves occupied a larger surface area ratio to the total surface area of the rat used compared to the anticipated device that will be used on the human.

\section{Electroporation and iontophoresis}

Electroporation (EP) is another method for enhancing the skin's permeability using an electroporator to induce temporal pores in the skin membrane. The pores on the skin of rabbits were induced followed by the application of different formulations of insulin [57]. Among all investigated formulations, insulin solution with EP had the best results when compared to insulin gel having penetration enhancers combined with EP.

Emulgel is a combined formulation of both emulsion and gel in a single dosage form that has received attention for topical formulation [58]. Emulgel was prepared by combining insulin with emu oil as a penetration enhancer and was examined on diabetic rabbits. BGLs decreased to $125 \pm 5 \mathrm{mg} / \mathrm{dl}$ from initially $250 \pm 10 \mathrm{mg} / \mathrm{dl}$ when the formulation is used in combination with iontophoresis. The later provided a synergic effect to the permeation enhancing the ability of the formulation.

\section{Microdermabrasion}

Microdermabrasion is known as a facial treatment and cosmetic resurfacing technique for the removal of fine lines, scars, and wrinkles. One study stated that microdermabrasion can be used for disrupting the dermal layer and enhancing the skin permeability for transdermal drug delivery [59]. An experiment was performed on diabetic hairless rats in which the skin was microdermabraded using a Gold Series MegaPeel machine to deliver insulin via transdermal reservoir patch. However, removing the stratum corneum using this technique did not increase skin permeability to insulin in comparison to microdermabrasion that removed full epidermis which provided the best results. According to the authors, full epidermis removal group had BGL reduction by $144 \mathrm{mg} / \mathrm{dL}$ in $3 \mathrm{~h}$ [59]. 


\section{Transdermal patches}

One of the latest approaches toward insulin delivery includes the application of smart patches. The patch contains glucose-responsive microneedles that release insulin automatically in response to the increase of the BGLs [60]. Each needle had a conical shape with a height of $600 \mu \mathrm{m}$, base radius of $150 \mu \mathrm{m}$ and a tip radius of about 10 $\mu \mathrm{m}$, which smaller than the smallest insulin needle, therefore ensuring painless administration. The painless microneedles hold glucose-responsive vesicles with an average diameter of $118 \mathrm{~nm}$. The vesicles are filled with insulin and glucose oxidase enzyme. The latter causes glucose oxidation in hyperglycemic conditions which triggers the dissociation of vesicles followed by insulin release. Streptozotocininduced type 1 diabetic mice treated with these patches containing 10 $\mathrm{mg} / \mathrm{kg}$ of insulin for each mouse showed a quick decline in BGL to nearly $200 \mathrm{mg} / \mathrm{dL}$ from approximately $550 \mathrm{mg} / \mathrm{dL}$ within $0.5 \mathrm{~h}$ and maintained a normoglycemic state for approximately $4 \mathrm{~h}$. The authors of the work claim that using these smart "closed-loop" patches in human can eliminate episodes of hyper and hypoglycaemia. Although this approach could really be all in one system (responding on demand to increases in glucose levels), it is early to be optimistic for a successful application to the human subject.

Also, transcutaneous delivery is possible through microneedle array patches composed of H2O2-responsive polymeric vesicles (PVS) similar to the one described above but differs in the polymeric materials used (fig. 3). The PVS are self-assembled from polyethylene glycol and phenylboronic ester (PBE)-conjugated polyserine forming a hollow structure loaded with insulin and glucose oxidase (GOx) [61]. The smart delivery of insulin is similar to that described by Yu et al., 2015 [60]. The release kinetics of insulin can be controlled by adjusting the concentration of GOx. The patches were applied to diabetic mice with an equivalent insulin dose of 10 $\mathrm{mg} / \mathrm{kg}$ for each mouse reduced glucose levels to $90 \mathrm{mg} / \mathrm{dL}$ in one hour and maintain it with normal ranges for nearly $5 \mathrm{~h}$, making it a well promising method for controlled non-invasive insulin delivery. Based on insulin dose used per $\mathrm{kg}$, it appears that a very large quantity of insulin would be needed if patches are to be applied in clinical trials. This can totally change the ability of the system to perform as well as when using mice model since insulin concentration and the surface area of the patch will have to be changed in a clinical application which can significantly influence insulin release kinetics. Furthermore, the duration for insulin release from the patches to maintain glucose levels was for only a few hours, when you expect to have days of therapy from traditional batches. Nevertheless, the concept of an integrated approach to having non-invasive insulin "smart" patches that could deliver insulin when needed is the kind of systems we should be looking for.

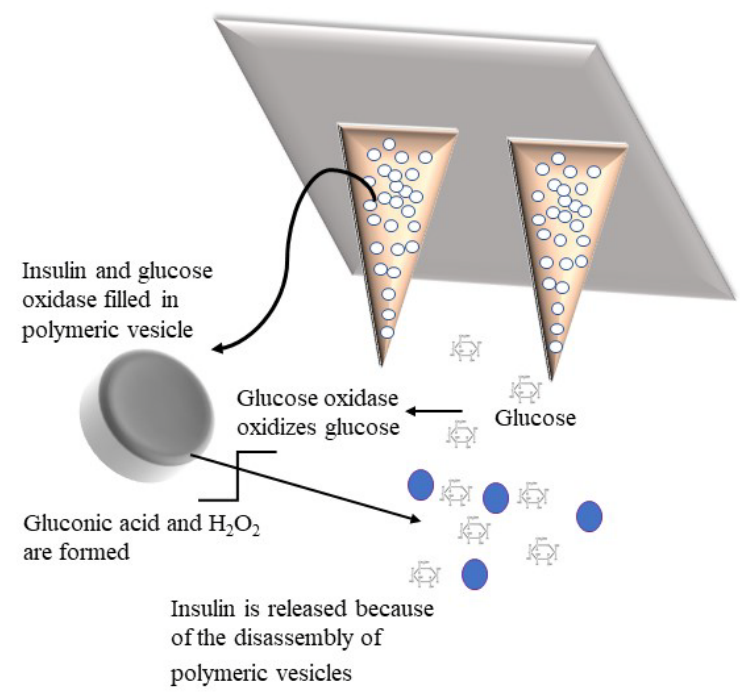

Fig. 3: Illustration showing the release process of insulin from a polymeric vesicle filled with insulin and glucose oxidase in the presence of glucose [60]
Pectin 'poly galacturonic acid' is known for the delivery of drugs to the colonic region of the gastrointestinal tract and for the sustained release of drugs [62]. Thus, a pectin insulin-containing dermal patch formulation can be explored for providing sustained transdermal delivery of insulin. The amidated pectin hydrogel insulin matrix patches containing insulin doses of 9.57, $16.80 \mu \mathrm{g} / \mathrm{kg}$ showed comparable glucose lowering to fast-acting insulin aspart administered subcutaneously in diabetic-induced rats, however, no sustained release was demonstrated from the investigation.

One transdermal patch for insulin delivery completed a clinical trial (NCT00519623) [63]. Altea Therapeutics was developing a transdermal patch called 'PassPort patch' that delivers insulin to the skin by creating micropores using bursts of thermal energy to produce small channels in the surface of the skin. The patch completed phase 1 and 2 for type 1 DM successfully and was well tolerated with no serious side effects except for mild temporal erythema on sites of application. The company was acquired by Nitto Denko Corporation (Japan) in 2012 and further development into clinical trial phase 3 was not sought for this insulin patch.

\section{Insulin jet injectors}

Powder needless injection (PNI) is an interesting transdermal delivery technique that uses high-speed gas flow to deliver powdered drugs into the skin without needles [64]. The aerodynamic properties of the device were enhanced by designing an automatic mechanical unit containing a valve system in addition to an enlarged de Laval nozzle. Two powder formulations of insulin were prepared as raw insulin and inorganic salt-carried insulin. The delivering efficiency and bioavailability were evaluated by experiments on rabbits, and it was found that phosphate-carried insulin powder had better results and higher hypoglycaemic effects compared to the raw insulin that was almost not effective when delivered by the PNI indicating the importance of the phosphate carrier in the delivery of insulin. In comparison to SC injection of phosphate-carried insulin solution, the PNI delivery efficiency was $72.25 \%$ as indicated by the area above the blood glucose curve (indicated glucose lowering effect). However, although the delivery of insulin is needless, the device might cause skin injuries if used improperly, Irritation caused by the device was related to many factors including the gas pressure and the nozzle distance and this needs to be optimized to be safe to use in practice. In principle, the idea is not new to the market, but the available devices use liquid insulin rather than powdered insulin. Their wide use has been hindered by cost, decreased absorption over the repeated administration, pain or bruising at the administration and the need to keep the product sterile to stay hygienic [48].

Glide Pharma developed a solid dose injector (SDI). The system uses a spring-powered handheld pen-like actuator to push a rod-like point end solid dosage forms [65]. Polysialic acid (PSA), a biodegradable natural polymer, has been used with insulin to form more soluble, stable and long circulatory half-life solution. In vitro and in vivo tests for SC delivery of solid PSA-insulin conjugate used Glide SDI, which was formulated to deliver 1 unit of insulin from a rod of $4 \mathrm{~mm}$ length. Three doses administered to each rat promoted a $100 \%$ penetration with delayed response throughout the first hour and prolonged efficacy that sustained for at least $5 \mathrm{~h}$, and upon comparison with PSAinsulin solution same hypoglycaemic effect was obtained.

\section{Rectal administration}

One study suggested rectal administration of insulin condensed dimple suppository since secretory degrative enzymes are much lower in the rectum than that of the stomach [66]. Insulin was condensed in a dimple with Labrasol ${ }^{\circledR}$ as absorption enhancer, then it was covered by sealing materials to direct its release toward rectal mucosa only and prevent insulin dilution by rectal fluid, hence offering a higher concentration gradient and improved absorption. In vivo tests, on male Wistar rats with suppositories administered into the rectum showed a glucose level reduction of maximum $22.9 \pm 3.2 \%$ at $30 \mathrm{~min}$ after administration compared to $8.2 \pm 5.8 \%$ when Labrasol ${ }^{\circledR}$ was not used.

Chitosan derivatives were also used analog to Labrasol ${ }^{\circledR}$ to enhance absorption and have been suggested for rectal as well as nasal administration. A study compared the effect of the degree of 
quaternization of $\mathrm{N}$-trimethyl chitosan chloride (TMC) and $\mathrm{pH}$ of the preparations on insulin absorption [67]. Different insulin $(4 \mathrm{IU} / \mathrm{ml})$ preparations were formulated with or without a $0.5 \% \mathrm{w} / \mathrm{v}$ of chitosan hydrochloride, TMC-L (12.3\% quaternization) and TMC-H (61.2\% quaternization) at $\mathrm{pH} 4.4$ and $\mathrm{pH}$ 7.4. Administration of solutions to rats rectally by a syringe showed that at $\mathrm{pH} 4.4$ a sharp increase in insulin levels peaking at $30 \mathrm{~min}$ after administration with blood glucose reduction by $38 \%, 22 \%$ and $22 \%$ for chitosan hydrochloride, TMC-H, and TMC-L respectively. In contrast, only TMC-H effectively increased insulin level to $\max$ at $22.5 \mathrm{~min}$ resulting in $29 \pm 7 \%$ blood glucose reduction at $\mathrm{pH}$ 7.4. The study demonstrated that not only the degree of quaternization is an important determining factor of insulin absorption, but also the insolubility of chitosan hydrochloride prevents it from being effective as absorption enhancers at $\mathrm{pH}$ 7.4.

\section{DISCUSSION}

Although extensive studies and efforts were carried out to have noninvasive alternatives to $\mathrm{SC}$ insulin injections, their existence is very limited as marketed products. An approximate representation of the current status and progress towards marketing approval of noninvasive insulin delivery systems is provided in fig. 4 . The analysis of published studies indicates that the majority of them are still in vitro and in vivo animal studies (about 91\%) with slim chances for progress into human trials. The percentages of non-invasive insulin delivery systems in various clinical trials do not exceed $6 \%$. It is even dubious that any of these systems will progress to the marketing approval stage. None of the studies in different clinical trial phases are considered an oral formulation of insulin for the primary purpose of lowering BGLs was successful so far.

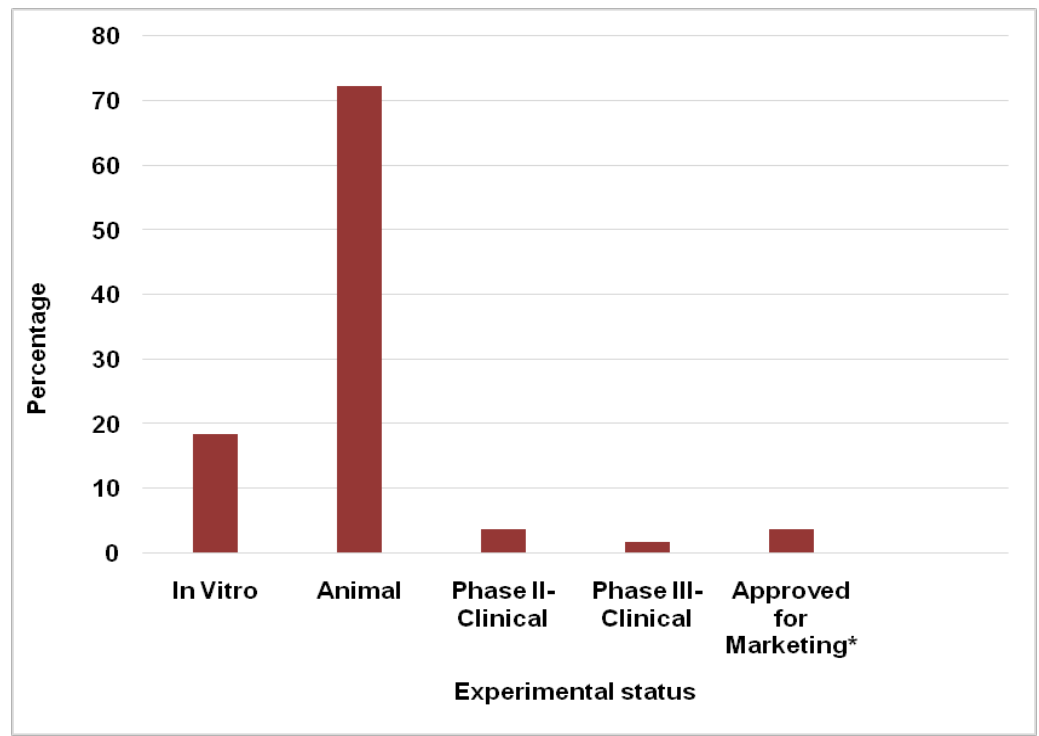

Fig. 4: Representation of current status of the development of non-invasive insulin delivery systems, ${ }^{*}$ Two of the three products were FDA approved

The issue of enzymatic degradation of insulin and its molecular weight impede the absorption of intact insulin molecules. The majority of the studies with oral administration of insulin attempted to improve bioavailability. In comparison with SC insulin injections, these improvements are not much promising since at best the results could show the relative bioavailability of up to $10 \%$. With low bioavailability, variability can become significant, preventing the utilization of any current oral formulation. For example, the HDV-I as clinical trials proved its beneficial effect in reducing BGLs postprandially upon coadministration with oral hypoglycaemic medications, however, it did not show dose-response correlation and the reduction was not significant enough for diabetic patients to have normal BGLs. This does not close the door for a futuristic smart pill technology. The idea of having HDV-I administered orally is tempting because unlike the lack of adequate glycaemic control of SC insulin injections due to marked peripheral hyperinsulinemia, the delivery of insulin directly to hepatocytes through the liver portal vein would allow better control of postprandial PGLs by directly toppling hepatic glucose production while increasing hepatic glucose uptake [13].

The marketing of Oral-Lyn ${ }^{\mathrm{m}}$ insulin formulation for buccal administration using "RapidMist" inhaler is only with limited success since it did not secure the marketing approval of the major authorities such as the US FDA and the European Medicines Agency (EMA). Oral-Lyn ${ }^{m+1}$ administration requires several puffs to deliver the dose along with poor bioavailability making it far from ideal for diabetic patients. The fast-acting liquid oral insulin is delivered from the metered-dose inhaler as fine aerosolized droplets into the buccal area for transmucosal absorption deliver with each puff delivering an intact unit of insulin each puff. The product is indicated for both types of DM along with other SC insulin preparation for further glycaemic control according to the Generex Biotechnology Corporation, the proprietor of the product. A window of opportunity still exists for buccal formulation development that is capable of enhancing absorption through permeability enhancers and also there is a need to prolong residence time for an adequate amount of insulin to be absorbed. Generex company had indicated that it is working on a formulation that allows the use of higher insulin concentration and improved formulation, but no outcomes have been published so far about the success of their attempt.

Exubera ${ }^{\circledR}$ was the first insulin inhalation product to receive FDA approval and consists of blisters containing human insulin inhalation powder, which are administered using the Exubera ${ }^{\circledR}$ Inhaler. The product proved to be a failure resulting in its withdrawal less than two years from its launch [34]. This was attributed to being expensive, cumbersome, flawed marketing, restriction in its use, poor bioavailability and accuracy of the delivered dose. Therefore, it was less favourable to both physicians and patients. Nevertheless, MannKind Corporation received FDA marketing approval relatively recently to commercialize insulin inhalation system Aferrza ${ }^{\circledR}$, having a compact size, unique as an "ultra-ultra" fast insulin, and improved bioavailability and dose flexibility in comparison to Exubera ${ }^{\circledR}$ [68]. The new product was crumbled by limiting factors despite its anticipated wider acceptance including adverse effects similar to Exubera ${ }^{\circledR}$ such as a decline in pulmonary function and unsubstantiated evidence of a slight increase in lung cancer incidence [52]. A further limiting factor to its sales is the need to assess patients' lungs and use spirometry prior to its prescription. The new insulin inhalation device Dance $501 \AA$ (Dance Biopharm Inc.) is currently in 
phase 2 trial using non-diabetic patients. The system features of dose individualization for each patient along with its handheld small size, employs liquid insulin that is expected to produce higher bioavailability than the DPIs (e. g. Exubera ${ }^{\circledR}$ and Aferrza $®$ ), with a lower incidence of coughing [53]. The fact that the second insulin inhalation product Afrezza ${ }^{\circledR}$ did not fulfill its promise in gaining popularity despite its advantages over Exubera ${ }^{\circledR}$ sheds a lot of doubts about the ability of Dance $501 \AA$ to perform differently. It is unclear how any future insulin inhalation system could address the needs of a certain diabetic population such as patients having chronic pulmonary diseases and smokers or eliminate the need of prescribers to assess patients' lungs functions before these products can be written in the prescription forms.

The focus on the transdermal delivery using "smart" patches could eliminate the need for monitoring BGLs as the systems themselves deliver bursts of insulin from specially designed vesicles whenever glucose levels rise as the case with prandial glucose and hence maintaining more consistent glucose levels. Therefore, such a system could be of real significance if they are successful in human by delivering therapeutic quantity of insulin sufficient for at least one day need without causing skin or other untoward effects. The results from published in vivo animal studies $[60,61]$ indicate that a lot of work to be done if these smart patches are to work on human subjects, mainly dealing with the amount of loaded insulin and the duration of insulin release to maintain desired BGLs for an appropriate length of time.

The fact that currently insulin needles are of large gauge number (28 to 31) make them almost painless to many patients. SC insulin injections are not only reliable with almost $100 \%$ bioavailability but enjoy a long history of success for both the prescribers and the patients to control hyperglycaemic conditions. It is unclear if any new non-invasive system will have such popularity in the next several years to come.

\section{CONCLUSION}

Non-invasive insulin delivery systems are offering diabetic patients with the alternative to the conventional SC injections. Unfortunately, none of the new systems were able to match the SC insulin in terms of bioavailability and cost. Because of their relatively short period in the market, they have not yet reached the expected popularity among patients and prescribing physicians. The search for ultimate insulin delivery systems is still challenging and hindered by the high cost, poor permeability and bioavailability, instability, adverse effects and the difference in diabetic patients' requirements. The most common route for administering drugs has been explored for delivering insulin orally, however, it is of dubious efficiency because of the inherent challenges associated with enzymatic and digestive juices, first pass metabolism and incomplete absorption. The different attempts to shield insulin and improve its uptake into the systemic circulation through the portal vein have only produced modest improvement in the insulin bioavailability and in many cases did not show linearity between the administered dose and the reduction in the BGLs. Therefore, the oral route remains an open area for improving insulin bioavailability, probably with systems that are capable of delivering insulin to hepatocytes. A nonorthodox approach to dealing with diabetes may be needed to eliminate the need for administering insulin altogether and reduce the healthassociated burden. Till then the SC insulin administration using microfine insulin needles to reduce associated pain will dominate insulin delivery systems for several years to come as indicated by the current products in different phases of clinical trials.

\section{ACKNOWLEDGMENT}

We would like to acknowledge the artistic work of the student Ms. Sarah Moawia Altabakha from Sama American Private School (Sharjah, UAE) for drawings representing Ora-Lyn ${ }^{\mathrm{TM}}$, Afrezza ${ }^{\circledR}$, Exubera ${ }^{\circledR}$ and Dance $501^{\circledR}$ insulin delivery systems.

\section{AUTHORS CONTRIBUTIONS}

All the author have contributed equally

\section{CONFLICTS OF INTERESTS}

Declared none

\section{REFERENCES}

1. Arya AK, Kumar L, Pokharia D, Tripathi K. Applications of nanotechnology in diabetes. Digest J Nanomaterials Biostructures 2008;3:221-5.

2. DA Kumar, V Kumar, V Tomer. Traditional medicinal systems for treatment of diabetes mellitus: a review. Int J Pharm Pharm Sci 2018;10:7-17.

3. AlKhamees OA, AlNemer KA, Bin Maneea MW, Al Sugair FA, AlEnizi BH, Alharf AA. Top 10 most used drugs in the Kingdom of Saudi Arabia 2010-2015. Saudi Pharm J 2018;26:211-6.

4. Shah R, Patel M, Maahs D, Shah V. Insulin delivery methods: past, present and future. Int J Pharm Investig 2016;6:1-9.

5. Tian W, Hu Q, Xu Y, Xuu Y. Effect of soybean-lecithin as an enhancer of buccal mucosa absorption of insulin. Biomed Mater Eng 2012;22:171-8.

6. Zhang L, Gao Z, Zhao X, Qi G. A natural lipopeptide of surfactin for oral delivery of insulin. Drug Delivery 2016;23:2084-93.

7. Chawla A, Chawla R, Jaggi S. Microvasular and macrovascular complications in diabetes mellitus: distinct or continuum? Indian J Endocrinol Metab 2016;20:546-51.

8. Shah D, Agrawal V, Parikh R. Noninvasive insulin delivery system: a review. Int J Appl Pharm 2010;2:35-40.

9. Kumar V, Choudhry I, Hurkat P, Jain A, Jain D. Oral insulin: myth or reality. Curr Diabetes Rev 2018;14:497-508.

10. Liu C, Shan W, Liu M, Zhu X, Xu J, Xu Y, et al. A novel ligand conjugated nanoparticles for oral insulin delivery. Drug Delivery 2016;23:2015-25.

11. Barbari GR, Dorkoosh FA, Amini M, Sharifzadeh M, Atyabi F, Balalaie $S$, et al. A novel nanoemulsion-based method to produce ultrasmall, water-dispersible nanoparticles from chitosan, surface modified with cell-penetrating peptide for oral delivery of proteins and peptides. Int J Nanomed 2017;12:3471-83.

12. Elsayed A, Al-Remawi M, Qinna N, Farouk A, Al-Sou'od KA Badwan AA. Chitosan-sodium lauryl sulfate nanoparticles as a carrier system for the in vivo delivery of oral insulin. AAPS PharmSciTech 2011;12:958-64.

13. Geho WB, Rosenberg LN, Schwartz SL, Lau JR, Gana TJ. A singleblind, placebo-controlled, dose-ranging trial of oral hepaticdirected vesicle insulin add-on to oral antidiabetic treatment in patients with type 2 diabetes mellitus. J Diabetes Sci Technol 2014;8:551-9.

14. Mahobia S, Bajpai J, Bajpai AK. An in vitro investigation of swelling controlled delivery of insulin from egg albumin nanocarriers. Iran J Pharm Res 2016;15:695-711.

15. Paul PK, Treetong A, Suedee R. Biomimetic insulin-imprinted polymer nanoparticles as a potential oral drug delivery system. Acta Pharm 2017;67:149-68.

16. He H, Lu Y, Qi J, Zhao W, Dong X, Wu W. Biomimetic thiamineand niacin-decorated liposomes for enhanced oral delivery of insulin. Acta Pharm Sin B 2018;8:97-105.

17. Mateti U, Rajakannan $\mathrm{T}$, Adla N, Valakkathala R. Insulin chewing gum: need of the day for diabetic patients. Int J Pharm Investig 2011;1:131-4

18. Cui M, Wu W, Hovgaard L, Lu Y, Chen D, Qi J. Liposomes containing cholesterol analogues of botanical origin as drug delivery systems to enhance the oral absorption of insulin. Int J Pharm 2015;489:277-84.

19. Niu M, Lu Y, Hovgaard L, Wu W. Liposomes containing glycocholate as potential oral insulin delivery systems: preparation, in vitro characterization, and improved protection against enzymatic degradation. Int J Nanomed 2011;6:1155-66.

20. Agrawal AK, Harde H, Thanki K, Jain S. Improved stability and antidiabetic potential of insulin containing folic acid functionalized polymer stabilized multilayered liposomes following oral administration. Biomacromolecules 2014;15:350-60.

21. Shofner JP, Phillips MA, Peppas NA. Cellular evaluation of synthesized insulin/transferrin bioconjugates for oral insulin delivery using intelligent complexation hydrogels. Macromol Biosci 2010;10:299-306.

22. Carr DA, Peppas NA. Assessment of poly(methacrylic acid-co-Nvinyl pyrrolidone) as a carrier for the oral delivery of therapeutic proteins using Caco-2 and HT29-MTX cell lines. J Biomed Mater Res A 2010;92:504-12. 
23. Mundargi RC, Rangaswamy V, Aminabhavi TM. Poly(Nvinylcaprolactam-co-methacrylic acid) hydrogel microparticles for oral insulin delivery. J Microencapsul 2011;28:384-94.

24. Si L, Zhao Y, Huang J, Li S, Zhai X, Li G. Calcium pectinate gel bead intended for oral protein delivery: preparation improvement and formulation development. Chem Pharm Bull 2009;57:663-7.

25. Pechenkin MA, Balabushevich NG, Zorov IN, Staroseltseva LK, Mikhalchik EV, Izumrudov VA, et al. Design, in vitro and in vivo characterization of chitosan-dextran sulfate microparticles for oral delivery of insulin. J Bioequivalence Bioavailability 2011;3:244-50.

26. Burger C, Maffezzolli S, Clasen P, Lucinda Silva RM, Rodrigues CA. Chitosan-alginate capsules as oral delivery system for insulin: studies in vitro and in vivo. Rev Ciencias Farm Basicase Appl 2008;29:239-44.

27. Lee H, Jeong C, Ghafoor K, Cho S, Park J. Oral delivery of insulin using chitosan capsules cross-linked with phytic acid. Biomed Mater Eng 2011;21:25-36.

28. Banerjee A, Lee J, Mitragotri S. Intestinal mucoadhesive devices for oral delivery of insulin. Bioeng Transl Med 2016;1:338-46.

29. Al-Tabakha MM, Issam Arida A, Fahelelbom KM, Sadek B, Abu Jarad RA. Performances of new generation of delayed-release capsules. J Young Pharm 2015;7:36-44.

30. Jindal SK, Singh M, Goswami M. Formulation and evaluation of insulin enteric microspheres for oral drug delivery. Acta Pharm Sci 2009;51:121-7.

31. Erel G, Kotmakçı M, Akbaba H, Sözer Karadaglı S, Kantarcı AG. Nanoencapsulated chitosan nanoparticles in emulsion-based oral delivery system: in vitro and in vivo evaluation of insulin-loaded formulation. J Drug Delivery Sci Technol 2016;36:161-7.

32. Kumria R, Goomber G. Emerging trends in insulin delivery: buccal route-review article. J Diabetol 2011;2:1-9.

33. Annabestani Z, Sharghi S, Shahbazi S, Sajad S, Karimi F, Taheri $\mathrm{E}$, et al. Insulin buccal spray (Oral-Lyn) efficacy in type 1 diabetes. Iran J Diabetes Lipid Disord 2010;9:1-4.

34. Adhikari SNR, Panda S. Buccal patches of atenolol formulated using fenugreek (Trigonella foenum-graecum L.) seed mucilage. Polim Med 2017;47:5-11.

35. Fröhlich E, Mercuri A, Wu S, Salar Behzadi S. Measurements of deposition, lung surface area and lung fluid for simulation of inhaled compounds. Front Pharmacol 2016;7:181.

36. Siekmeier R, Scheuch G. Inhaled insulin-does it become reality? J Physiol Pharmacol 2008;59 Suppl 6:81-113.

37. Srinivasan G, Shetty A. Advancements in a dry powder inhaler. Asian J Pharm Clin Res 2017;10:8-12.

38. Lim SH, Park HW, Shin CH, Kwon JH, Kim CW. Human insulin microcrystals with lactose carriers for pulmonary delivery. Biosci Biotechnol Biochem 2009;73:2576-82.

39. Rodrigues S, Cardoso L, da Costa AMR, Grenha A. Biocompatibility and stability of polysaccharide polyelectrolyte complexes aimed at respiratory delivery. Materials (Basel) 2015;8:5647-70.

40. Nema T, Jain A, Jain A, Shilpi S, Gulbake A, Hurkat P, et al. Insulin delivery through nasal route using thiolated microspheres. Drug Delivery 2013;20:210-5.

41. Hamad IM, Arida AI, Al-Tabakha MM. Effect of the lubricant magnesium stearate on changes of specific surface area of directly compressible powders under compression. Jordan J Pharm Sci 2015;8:23-35.

42. Tanaka A, Furubayashi T, Matsushita A, Inoue D, Kimura S, Katsumi $\mathrm{H}$, et al. Nasal absorption of macromolecules from powder formulations and effects of sodium carboxymethyl cellulose on their absorption. PLoS One 2016;11:1-11.

43. Pillion DJ, Fyrberg MD, Meezan E. Nasal absorption of mixtures of fast-acting and long-acting insulins. Int $\mathrm{J}$ Pharm 2010;388:202-8.

44. Khafagy ES, Kamei N, Nielsen EJB, Nishio R, Takeda Morishita M. One-month subchronic toxicity study of cell-penetrating peptides for insulin nasal delivery in rats. Eur J Pharm Biopharm 2013;85(3, Pt A):736-43.

45. Kuehl PJ, Cherrington A, Dobry DE, Edgerton D, Friesen DT, Hobbs C, et al. Biologic comparison of inhaled insulin formulations: Exubera ${ }^{\mathrm{TM}}$ and novel spray-dried engineered particles of dextran-10. AAPS PharmSciTech 2014;15:1545-50.
46. Agrawal AK, Gupta PN, Khanna A, Sharma RK, Chandrabanshi HK, Gupta N, et al. Development and characterization of in situ gel system for nasal insulin delivery. Pharmazie 2010;65:188-93.

47. Patel A, Cholkar K, Mitra AK. Recent developments in protein and peptide parenteral delivery approaches. Ther Delivery 2014;5:337-65.

48. Al-Tabakha M, Arida A. Recent challenges in insulin delivery systems: a review. Indian J Pharm Sci 2008;70:278-86.

49. Laube BL. Aerosolized medications for gene and peptide therapy. Respir Care 2015;60:806-24.

50. Boss AH, Petrucci R, Lorber D. Coverage of prandial insulin requirements by means of an ultra-rapid-acting inhaled insulin. J Diabetes Sci Technol 2012;6:773-9.

51. Al-Tabakha MM. Future prospect of insulin inhalation for diabetic patients: the case of Afrezza versus exubera. J Controlled Release 2015;215:25-38.

52. Pettus J, Santos Cavaiola T, Edelman SV. Recommendations for initiating use of Afrezza inhaled insulin in individuals with type 1 diabetes. Diabetes Technol Ther 2018;20:448-51.

53. Fink JB, Molloy L, Patton JS, Galindo-Filho VC, de Melo Barcelar J, Alcoforado L, et al. Good things in small packages: an innovative delivery approach for inhaled insulin. Pharm Res 2017;34:2568-78.

54. Jabbari N, Asghari MH, Ahmadian H, Mikaili P. Developing a commercial air ultrasonic ceramic transducer to transdermal insulin delivery physical characteristics of ultrasound. J Med Signal Sens 2015;5:117-22.

55. Teo SY, Lee SY, Rathbone MJ, Gan SN. Polymeric materials as platforms for topical drug delivery: a review. Int J Pharm Pharm Sci 2017;9:14-20.

56. Banerjee A, Ibsen K, Iwao Y, Zakrewsky M, Mitragotri S Transdermal protein delivery using choline and geranate (CAGE) deep eutectic solvent. Adv Healthcare Mater 2017;6:1-11.

57. Mohammad EA, Elshemey WM, Elsayed AA, Abd-Elghany AA Electroporation parameters for successful transdermal delivery of insulin. Am J Ther 2016;23:e1560-7.

58. Akram M, Naqvi SBS, Khan A. Design and development of insulin emulgel formulation for transdermal drug delivery and its evaluation. Pak J Pharm Sci 2013;26:323-32.

59. Andrews S, Lee JW, Choi SO, Prausnitz MR. Transdermal insulin delivery using microdermabrasion. Pharm Res 2011;28:2110-8.

60. Yu J, Zhang Y, Ye Y, DiSanto R, Sun W, Ranson D, et al. Microneedle-array patches loaded with hypoxia-sensitive vesicles provide fast glucose-responsive insulin delivery. Proc Proc Natl Acad Sci U S A 2015;112:8260-5.

61. $\mathrm{Hu} \mathrm{X}, \mathrm{Yu}$ J, Qian $\mathrm{C}, \mathrm{Lu} \mathrm{Y,} \mathrm{Kahkoska} \mathrm{AR,} \mathrm{Xie} \mathrm{Z,} \mathrm{et} \mathrm{al.} \mathrm{H}_{2} \mathrm{O}_{2}$ responsive vesicles integrated with transcutaneous patches for glucose-mediated insulin delivery. ACS Nano 2017;11:613-20.

62. Hadebe SI, Ngubane PS, Serumula MR, Musabayane CT Transdermal delivery of insulin by amidated pectin hydrogel matrix patch in streptozotocin-induced diabetic rats: effects on some selected metabolic parameters. PLoS One 2014;9:e101461.

63. Altea Therapeutics. Transdermal basal insulin patch study in type 1 diabetes-study results. Clinical Trials; 2010. p. NCT00519623. Available from: https://clinicaltrials.gov/ ct2/show/results/NCT00519623?term=NCT00519623andran $\mathrm{k}=1$. [Last accessed on $18 \mathrm{Jul} 2018$ ]

64. Li C, Wang Z, Tu C, Wang J, Jiang B, Li Q, et al. Needle-free injection of insulin powder: delivery efficiency and skin irritation assessment. J Zhejiang Univ Sci B 2014;15:888-99.

65. Zhang R, Jain S, Rowland M, Hussain N, Agarwal M, Gregoriadis G. Development and testing of solid dose formulations containing polysialic acid insulin conjugate: next generation of long-acting insulin. J Diabetes Sci Technol 2010;4:532-9.

66. Matsumoto A, Murakami K, Watanabe C, Murakami M. Improved systemic delivery of insulin by condensed drug loading in a dimpled suppository. Drug Discovery Ther 2017;11:293-9.

67. Du Plessis LH, Kotze AF, Junginger HE. Nasal and rectal delivery of insulin with chitosan and N-trimethyl chitosan chloride. Drug Delivery 2010;17:399-407.

68. Muchmore DB. The need for faster insulin. J Diabetes Sci Technol 2017;11:157-9. 